\title{
Dietary diversity is inversely correlated with pre-pregnancy body mass index among women in a Michigan pregnancy cohort
}

\author{
Breanna M. Kornatowski ${ }^{1}$, Sarah S. Comstock ${ }^{\text {Corresp. } 1}$ \\ ${ }^{1}$ Department of Food Science and Human Nutrition, Michigan State University, East Lansing, Michigan, United States \\ Corresponding Author: Sarah S. Comstock \\ Email address: comsto37@msu.edu
}

Background. During pregnancy, healthy maternal body weight and a nutritionally complete diet provide a favorable environment for fetal development. Yet nearly twothirds of women of reproductive age in the United States (US) are either overweight or obese. The objective of this research was to investigate the relationship between a measure of dietary diversity and body mass index (BMI) in a sample of women enrolled in a US pregnancy cohort. Methods. Dietary data was obtained from one 24-hour dietary recall collected during the third trimester of pregnancy $(n=40)$. Pre-pregnancy BMI was calculated from pre-pregnancy weight and height self-reported by survey at the time of enrollment. Using the Minimum Dietary Diversity for Women (MDD-W) indicator developed by the Food and Agriculture Organization of the United Nations, dietary data was categorized and scored. Results. Overall, 35\% of participants did not achieve minimum dietary diversity (MDD-W $\geq 5$ ). In addition, $45 \%$ of participants were obese, $35 \%$ were normal weight and $20 \%$ were overweight. Women with higher BMI had lower MDD-W scores than women with lower BMI $(p<0.05)$. The median MDD-W for both normal and overweight women was $\geq 5$ indicating that normal and overweight pregnant women tended to consume a diet that was high in micronutrients. Conversely, the median MDD-W for obese women was below 5 meaning these women tended to consume a diet that was low in micronutrient density. The most commonly consumed food group was grain. In addition, $75 \%$ of all participants consumed sweetened drinks. Individuals with an MDD-W score $\geq 5$, were more likely to have consumed dark green leafy vegetables, vitamin A-rich fruits or vegetables, other vegetables and other fruits than those with MDD-W scores $<5$. Discussion. In this study, we show that a food group diversity indicator that has been shown to reflect adequacy of micronutrient intake in populations from less economically developed countries may also be informative in US populations. Furthermore, these results reflect the importance of encouraging all pregnant women with less varied diets to consume more fruits and vegetables. 
1

2

3

4

5

6

7

8

9

10

11

12

13

14

15

16

17

18

19

20

21

22

23

24

25

26

27

28

29

30

31

32

33

34

35

36

37

38

39

40

41

42
Dietary Diversity is Inversely Correlated with Pre-Pregnancy Body Mass Index among Women in a Michigan Pregnancy Cohort

Breanna M. Kornatowski and Sarah S. Comstock

Department of Food Science and Human Nutrition Michigan State University $139 \mathrm{C}$ Trout 469 Wilson Rd

East Lansing, Michigan 48824

United States of America

Corresponding Author: Sarah Comstock, Email: comsto37@msu.edu 
43 List of Abbreviations

$44 \quad$ - BMl: body mass index.

45 - FAO: Food and Agriculture Organization.

46 - MDD-W: Minimum Dietary Diversity for Women of Reproductive Age.

47 - SAS: Statistical Analysis Software.

48 
49

50

51

52

53

54

55

56

57

58

59

60

61

62

63

64

65

66

67

68

69

70

71

Abstract

Background. During pregnancy, healthy maternal body weight and a nutritionally complete diet provide a favorable environment for fetal development. Yet nearly twothirds of women of reproductive age in the United States (US) are either overweight or obese. The objective of this research was to investigate the relationship between a measure of dietary diversity and body mass index (BMI) in a sample of women enrolled in a US pregnancy cohort.

Methods. Dietary data was obtained from one 24-hour dietary recall collected during the third trimester of pregnancy $(n=40)$. Pre-pregnancy BMI was calculated from prepregnancy weight and height self-reported by survey at the time of enrollment. Using the Minimum Dietary Diversity for Women (MDD-W) indicator developed by the Food and Agriculture Organization of the United Nations, dietary data was categorized and scored.

Results. Overall, $35 \%$ of participants did not achieve minimum dietary diversity (MDDW $\geq 5$ ). In addition, $45 \%$ of participants were obese, $35 \%$ were normal weight and $20 \%$ were overweight. Women with higher BMI had lower MDD-W scores than women with lower BMI $(p<0.05)$. The median MDD-W for both normal and overweight women was $\geq 5$ indicating that normal and overweight pregnant women tended to consume a diet that was high in micronutrients. Conversely, the median MDD-W for obese women was below 5 meaning these women tended to consume a diet that was low in micronutrient density. The most commonly consumed food group was grain. In addition, $75 \%$ of all participants consumed sweetened drinks. Individuals with an MDD-W score $\geq 5$, were 
72 more likely to have consumed dark green leafy vegetables, vitamin A-rich fruits or

73 vegetables, other vegetables and other fruits than those with MDD-W scores $<5$.

74 Discussion. In this study, we show that a food group diversity indicator that has been

75 shown to reflect adequacy of micronutrient intake in populations from less economically

76 developed countries may also be informative in US populations. Furthermore, these

77 results reflect the importance of encouraging all pregnant women with less varied diets

78 to consume more fruits and vegetables. 
79 Introduction

80 One in four American women are obese prior to becoming pregnant (Branum et

81 al. 2016). Obesity is associated with numerous health issues (Williams et al. 2015),

82 especially in pregnancy. In fact, an unhealthy body mass index (BMI) and diet in

83 pregnancy exerts negative effects on the developing fetus and leads to health problems

84 for the child later in life (Godfrey et al. 2017; Poston et al. 2016). Furthermore, women

85 who are obese are more likely to have children who are obese (Oestreich \& Moley

86 2017), perpetuating health problems from generation to generation. There is evidence

87 that obese individuals are deficient in micronutrients (Costa et al. 2018; Krzizek et al.

88 2017; Stankowiak-Kulpa et al. 2017).

89 Women of reproductive age require a more nutrient-dense diet because of the 90 physiological needs during pregnancy (Gernand et al. 2016). Commonly, women of

91 reproductive age consume supplemental micronutrients including iron, folic acid, and 92 zinc (Gernand et al. 2016). An insufficient amount of micronutrients during pregnancy

93 can create complications such as congenital malformations in the baby and increased 94 risk of hemorrhage during delivery (Black 2001). In addition, insufficient micronutrients 95 can inhibit physical, neurobehavioral, and immunological growth among other 96 complications (Gernand et al. 2016; Ojha et al. 2013; Sullivan et al. 2009; Zerfu \& Ayele 97 2013). Consuming prenatal vitamins can provide these micronutrients, however, the 98 "most desirable" way to meet micronutrient needs is through a "sustained diet of various 99 micronutrient-dense foods" (Gernand et al. 2016; Hanson et al. 2015). Unfortunately, 100 only around 78 percent of pregnant women in the United States recently reported taking 
101 some sort of multivitamin while they were pregnant, making it even more important for

102 women to consume micronutrients through their diet (Sullivan et al. 2009).

Although it is known that micronutrient intake is important in pregnancy and that obesity in pregnancy can lead to negative birth outcomes, the relationship between pre-

excellent way to determine micronutrient status of a population is to apply the Food and

diversity is not only a predictor for micronutrient status and diet quality, but it is also a

predictor of obesity. In a study done by Wolongevicz et al., women with lower diet

quality were significantly more likely to be overweight or obese compared to women

with higher diet quality (Wolongevicz et al. 2010). on pregnancy outcomes and child health, little research has examined the potential association between pre-pregnancy BMI and dietary diversity in pregnancy . A diet of high micronutrient adequacy can be achieved through a diverse diet that includes many different food groups. The minimum dietary diversity for women (MDD-W) indicator assesses micronutrient adequacy by determining whether a subject consumed at least

118 five out of ten categories of food groups in the past day (Martin-Prevel et al. 2015). A 119 score of $\geq 5$ indicates the individual has a diet of an estimated micronutrient adequacy

120 and has a diverse diet. In a given population, the proportion of women who achieve 121 dietary diversity can be used as an indicator for higher micronutrient adequacy (FAO \& 122360 2016). The objective for this study was to measure diversity in dietary intake by 123 women during their third trimester of pregnancy and to determine if MDD-W score was 
124 associated with pre-pregnancy BMI. The hypothesis for this study was that obese

125 women would have lower MDD-W scores than women of normal or healthy weight,

126 meaning obese women have diets with a lower estimated micronutrient adequacy.

127

128 Materials and Methods

129 Participants

130 This study used data from two studies, one study was nested in the Archive for

131 Research on Child Health (ARCH) called ARCH GUT and the other study was a stand

132 alone study called BABY GUT. The ARCH study is a cohort assembled in pregnancy to

133 examine relationships between exposures assessed prospectively in pregnancy and the

134 health and development of children. A total of 1,043 women were enrolled in the ARCH

135 cohort from 2008-2017 with 53 women enrolled in ARCH during the time ARCH GUT

136 was recruiting (Nov 2015-2017). ARCH exclusion criteria were age $<18$ years and

137 inability to answer a questionnaire in English. ARCH women were recruited at their first

138 prenatal visit (mean gestational age at enrollment, 13.4 weeks); were briefly interviewed

139 about nutrition, physical activity, depression and socio-economic status; consented to

140 the archiving of three urine specimens and extra blood obtained at two clinically-

141 required phlebotomies; allowed access to birth certificates, medical records and the

142 state-archived newborn blood spot; and consented to postnatal follow-up. BABY GUT

143 recruitment relied on responses to flyers placed in several OB/GYN clinics in the

144 Lansing, Ml area. Exclusion criteria were as in $\mathrm{ARCH}$, but also excluded women with

$145 \mathrm{BMI}<18.5$. Women could enroll at any point in pregnancy, but data for this study were

146 collected during the third trimester. All participants provided written informed consent. 
147 Study procedures for ARCH (IRB\#C07-1201), ARCH GUT (IRB\# 14-170M) and BABY

148 GUT (IRB\# 15-1240) were approved by the Michigan State University IRB.

149 Data presented here were collected from 40 pregnant women who were enrolled

150 in either ARCH GUT $(n=25)$ or BABY GUT $(n=15)$ in the Lansing and Traverse City

151 areas of the state of Michigan, USA. All 40 participants completed self-administered

152 questionnaires that included an open recall of dietary intake over a 24-hour period.

153 Participants were asked to report age, pre-pregnancy weight, height, and parity. The

154 validity of self-reported pre-pregnancy weight and height to determine pre-pregnancy

155 weight status classification has been confirmed previously (Shin et al. 2014). Participant 156 characteristics are shown in Table 1.

157

158 Diet Diversity Analysis

159 The 24-hour dietary recall data was used to determine which of the 22 MDD-W

160 categories (FAO \& 360 2016) a participant had consumed. These 22 categories

161 included: grains; white roots and tubers, and plantains; pulses (beans, peas and lentils);

162 nuts and seeds; milk and milk products; organ meat; meat and poultry; fish and

163 seafood; eggs; dark green leafy vegetables; vitamin A-rich vegetables, roots and tubers;

164 vitamin A-rich fruits; other vegetables; other fruits; insects and other small protein foods;

165 red palm oil; other oils and fats; savory and fried snacks; sweets; sugar-sweetened

166 beverages; condiments and seasonings; other beverages and foods. A subset (10

167 groups, Table 2) of the 22 categories were used to compute the MDD-W as described

168 (FAO \& 360 2016). The MDD-W is a dichotomous scale with a cut-off based on

169 consumption of at least 5 of the following 10 food groups: (1) Grains, roots, and tubers; 
170 (2) Pulses; (3) Nuts and seeds; (4) Dairy; (5) Meat, poultry, and fish; (6) Eggs; (7) Dark

171 leafy green vegetables; (8) Other Vitamin-A rich fruits and vegetables; (9) Other

172 vegetables; and (10) Other fruits. A subset of 3 groups (savory and fried snacks,

173 sweets, sugar-sweetened beverages) was used to assess intake of low-nutrient-density

174 foods. Briefly, if a woman consumed one or more items in a category, she received a

175 point for that category. For example, if a woman consumed cheese (dairy), milk (dairy),

176 eggs (eggs), and spinach (dark green leafy vegetables), she would receive one point for

177 dairy, one point for eggs and one point for dark green leafy vegetables resulting in a

178 final score of 3. Two researchers independently scored dietary intake data, and there

179 were no disagreements in scoring results. No registered dietitians were involved in this

180 project. Participants with scores of 5 or above were considered to have achieved

181 minimum dietary diversity, based on the dichotomous scoring rubric (FAO \& 360 2016).

182 Women were assigned a BMI category using the United States Centers for Disease

183 Control definitions (Garrow \& Webster 1985; 1998): normal or healthy weight (18.5 $\leq$

$184 \mathrm{BMI}<25)$, overweight $(25 \leq \mathrm{BMI}<30)$, obese $(\mathrm{BMI} \geq 30)$.

Statistical Analyses

187

Fisher's exact test was used to compare ARCH GUT and BABY GUT participant 188 characteristics as well as to compare among groupings within participant characteristics

189 (such as BMI, age, etc.) for all participants. Fisher's exact test was also used to 190 compare the percent of individuals consuming a specific food category between those who achieved MDD-W scores of $\geq 5$ versus those who did not. Spearman rank

192 correlation was used to assess the association between total MDD-W score and BMI. 
193 Fisher's exact test was used to compare the percent of individuals achieving a MDD-W

194 score $\geq 5$ across the three BMI categories. Kruskal-Wallis was used to compare total

195 MDD-W scores across BMI categories followed by pairwise comparisons using the

196 Dwass, Steel, Critchlow-Fligner multiple comparison procedure. All statistical analyses

197 were conducted with SAS version 9.4 (Cary, NC).

198

199 Results

200 Participant Characteristics

201 Participant characteristics did not differ between ARCH GUT and BABY GUT

202 (Table 1). There was a trend for more ARCH GUT participants to be obese compared to 203 BABY GUT participants $(p=0.104)$. Most of the women in both ARCH GUT and BABY 204 GUT were between the ages of 30 and 39 years. Few women in either study had given 205 birth to more than two children.

206 To calculate an MDD-W score for each participant, consumption of 10 food

207 groups was assessed. Those pregnant women with MDD-W scores $\geq 5$ more commonly

208 consumed vegetables and fruits than did women with MDD-W scores <5 (Table 2).

209 Overall, 35\% of participants did not achieve minimum dietary diversity. All participants,

210 with the exception of one, consumed grains. The majority of participants also consumed

211 dairy. Pulses such as beans, peas, and lentils were consumed the least. None of the

212 participants with low dietary diversity consumed pulses, and only $19.2 \%$ of participants

213 achieving minimum dietary diversity consumed them. Meat, fish and poultry were more

214 commonly consumed than eggs. 
216

217

218

219

220

221

222

223

224

225

226

227

228

229

230

231

232

233

234

235

236

237 populations.
MDD-W Score and BMI

Third trimester MDD-W score and maternal pre-pregnancy BMI were inversely correlated (Figure 1). Most normal weight women (86\%) and overweight women (88\%) achieved minimum dietary diversity with an MDD-W score greater than or equal to 5 (Figure 2). Conversely, only $39 \%$ of obese women achieved minimum dietary diversity. Significantly fewer obese women achieved minimum dietary diversity compared to normal weight women $(p=0.012)$ and compared to overweight women $(p=0.036)$.

Furthermore, third trimester MDD-W scores differed by pre-pregnancy BMI category, however, no pairwise comparisons were significant (Figure 3). The median MDD-W total for obese women was under 5, while the median MDD-W for overweight and normal weight women was 5 or above.

\section{Low Nutritional Density Food Consumption}

Figure 4 represents the consumption of three low nutrient density food groups including, savory snacks, sweets, and sweetened drinks. Although no comparisons across BMI categories or study cohort were statistically significant, numerically, a higher percentage of BABY GUT participants consumed all three low nutrient density food categories than ARCH GUT participants. Three-quarters of all participants consumed sweetened drinks. The MDD-W protocol defines sweetened drinks as beverages such as fruit juices, sodas, chocolate drinks, sweet teas, coffee with sugar, and energy drinks. Overall, consumption of foods with low nutritional density was high in the study 238 
240

241

242

243

244

245

246

247

248

249

250

251

252

253

254

255

256

257

258

259

260

261

Discussion

In this study, pre-pregnancy BMI was significantly inversely associated with dietary diversity during the third trimester of pregnancy. Fewer women who were obese prior to becoming pregnant reached minimum dietary diversity. Furthermore, women who failed to meet minimum dietary diversity were less likely to consume fruits and vegetables than women who achieved minimum dietary diversity. Low nutrient density foods were consumed frequently by all participating pregnant women.

The result that dietary diversity was inversely correlated with BMI in these pregnant women is similar to results obtained in other studies of non-pregnant women of a similar age. In a study of females 18-28 years of age, it was found that there was an inverse association between dietary diversity score (calculated based on intake of five main food groups and 25 sub-groups) and obesity (Azadbakht \& Esmaillzadeh 2011).

Dietary assessment based on dietary patterns is common in nutritional epidemiology (Hu 2002). In fact, various methods of assessing dietary patterns result in common conclusions from different data sets (Liese et al. 2015). However, the MDD-W score, a validated method of assessing dietary intake at the population level, minimizes participant and researcher burden. Furthermore, the results from the current study mirror those in other studies focused on dietary patterns. For instance, all three dietary patterns that emerged in a study of obese pregnant women living in the United Kingdom were characterized by consumption of poor diets (Flynn et al. 2015). Using methods that did not rely on a measure of dietary diversity, Mohd-Shurki et al. found that obese, 
262 pregnant women tended to have a diet that was energy-rich but low in micronutrient

263 density (Mohd-Shukri et al. 2015). In four of five low and middle income countries

264 (Bangladesh, Mali, Mozambique, Burkina Faso, and the Philippines) few women

265 achieved dietary diversity, and the prevalence of nutrient adequacy was below 50

266 percent in pregnant women and even lower among lactating women (Arimond et al.

267 2010). Studies using the Healthy Eating Index have also found an association between

268 higher pre-pregnancy BMI and poor dietary intake among pregnant women (Laraia et al.

269 2007; Shapiro et al. 2016; Shin et al. 2016; Tsigga et al. 2011) as has other research

270 assessing dietary quality in pregnancy. The dietary data collected in this study could not

271 be used to calculate the Health Eating Index because there was no information about

272 the amount of food consumed.

273 Over 60 percent of the pregnant women enrolled in the ARCH GUT and BABY

274 GUT studies achieved minimum dietary diversity. This is in stark contrast to similar

275 research conducted in low- and middle-income countries. In Kenya, only 15 percent of

276 the pregnant women, ages 16 to 49 years, achieved dietary diversity (Kiboi et al. 2017).

277 The Kenyan study used a slightly more specific diversity score that included 14 food

278 groups instead of 10 . However, the FAO had developed the 14-group score and

279 participant scores were based on a 24-hour recall making this study similar to those

280 using the MDD-W. In a systematic assessment of 187 countries, 54 percent of women

281 achieved dietary diversity. MDD-W was not used in that study, however, a similar diet

282 scale was used based on consumption of ten food groups ascertained from a 24-hour

283 recall (Imamura et al. 2015). Combined, these results support the importance of

284 considering the geographic location when interpreting results from similar studies and 
285 suggest that grouping large populations of women from different geographical areas into 286 a single statistic may be misleading.

Lack of dietary diversity is more commonly considered an issue for women living 288 in low and middle income nations (Chakona \& Shackleton 2017). Our research 289 demonstrates that a lack of dietary diversity is also an issue in the United States. One 290 reason cited for lack of dietary diversity is a lack of access to diverse food sources 291 (Imamura et al. 2015). In our study, food access was not measured so it's not clear if a 292 lack of access to a variety of foods contributed to the low DDW scores in obese women. 293 Other research has demonstrated that individuals living in areas with poor access to 294 healthy foods are more likely to be obese (Chi et al. 2013; Vargas et al. 2017), so there 295 is a possibility that obesity is a proxy measure for living in a food desert. Providing 296 pregnant women with a door-step delivery of a diverse array of foods is one potential 297 way to improve dietary diversity. This would combat common barriers to intake including 298 poor availability of foods locally, challenges in shopping for healthy foods, time 299 limitations of preparing healthy foods, and lack of knowledge or motivation to buy foods 300 that are unfamiliar. The current study did not collect data to ascertain participants' proximity to a food desert or access to food. Future studies should account for this 302 possibility. In our study, regardless of pre-pregnancy BMI status, fruits and vegetables were 304 the foods most commonly missing from the diets of women with low MDD-W scores. 305 However, low-nutrient foods were commonly consumed. It could be that low-nutrient 306 density foods are displacing fruits and vegetables from the diets of our participants. This 307 is consistent with other reports of dietary intake among adults in the US (DHHS \& USDA 
308 2015).Furthermore, children born to women who consumed the most fruits during

309 pregnancy performed better on cognitive tests at 1 year of age (Bolduc et al. 2016) and

310 a meta-analysis concluded maternal diet quality during pregnancy is positively

311 associated with child neurodevelopment and cognitive development (Borge et al. 2017).

312 Others report that maternal fruit and vegetable consumption increases infant head

313 circumference (Loy et al. 2011). Given these associations and that fruit and vegetable

314 consumption has been associated with numerous health benefits, this low intake of

315 fruits and vegetables by pregnant women is concerning.

316 As briefly mentioned above, a large proportion of the pregnant women in this

317 study consumed low nutrient density foods. It was surprising that this intake did not

318 differ by pre-pregnancy BMI category, but this is consistent with other reports in the US

319 (DHHS \& USDA 2015). For instance, three-quarters of women in ARCH GUT and BABY

320 GUT reported consuming at least one sweetened drink in their 24-hour recall. This is

321 consistent with the 2011-2014 NHANES report that 68 percent of adults living in the

322 Northeast United States consumed at least one sweetened drink each day and 40

323 percent of all women in the US consume sweetened beverages daily (Park et al. 2015).

324 These results are important as sweetened beverage consumption is a significant

325 contributing factor to the rise in overweight and obesity as well as associated with a

326 higher risk of hypertension, congenital heart disease, preterm delivery, and gestational

327 diabetes (Englund-Ogge et al. 2012; Malik et al. 2006; Marquez 2012; Xi et al. 2015).

328 Despite consistency in intakes of low nutrient density foods across pre-pregnancy BMI

329 categories, risk for diseases associated with such intake are only reported for obese

330 women. It is likely that, although there is intake of these foods by all women, women in 
331 some BMI categories consume larger volumes of such foods. Unfortunately, the

332 methods used in this research did not assess amounts of food consumed.

There were additional limitations of this research. The study included a modest

334 sample size $(n=40)$ and relied on a single self-reported 24-hour dietary recall. A single

335 recall is not representative of long-term dietary intake. However, the MDD-W is intended

336 to be used with single dietary recalls as it is applicable for use with groups of individuals

337 (in this instance: groups of normal weight, overweight and obese women) (FAO \& 360

2016). The MDD-W is not meant to be used to describe characteristics of individual

women. No information about dietary supplement intake or prenatal vitamin

consumption was collected from participants. The study population had a higher rate of

obesity (45\%) compared to those of the state of Michigan (32.5\%, all adults ((Prevention

2018)) and United States (25\%, women prior to pregnancy((Prevention 2018)). The

cause of this discrepancy is unknown, but may be related to the method of advertising

for the studies. Study recruitment flyers stated, "You can help us learn if there are ways

to prevent childhood tummy problems and obesity." Therefore, women who were obese might have been more likely to participate. Furthermore, this sample is a collection of participants recruited into two separate cohorts. However, all data used in this analysis was collected using the same survey instruments and was collated by the same research staff. Finally, the study was conducted within a geographically limited (one urban and one rural area in Michigan) population. pregnancy and pre-pregnancy BMI in a larger, more representative study population to confirm the inverse relationship between dietary diversity and pre-pregnancy BMI holds 
354 in a number of geographical locations across the United States. In addition, future

355 research could explore these connections stratified not only by geographical location

356 but also by access to food, socioeconomic status or parental education level.

357

358 Conclusions

359 Based on a single 24-hour dietary recall, women who were obese prior to 360 becoming pregnant failed to consume a diverse diet during the third trimester of

361 pregnancy. Furthermore, pregnant women who failed to achieve minimum dietary

362 diversity $(M-D D W<5)$ in their third trimester of pregnancy were less likely to consume

363 fruits and vegetables than women who achieved minimum dietary diversity. Diverse

364 dietary intake not only ensures adequate vitamin and mineral intake but also increases

365 fiber and phytochemical intake. Despite being important for human health and a healthy

366 pregnancy, neither fiber nor phytochemicals are found in most prenatal vitamins. Thus,

367 prenatal care providers should emphasize the necessity of consuming a diverse diet

368 when providing nutrition information to pregnant women. Given the results of this

369 research, this recommendation is especially relevant for pregnant women who were

370 obese prior to becoming pregnant, a population has been demonstrated to be open to

371 behavior change with respect to pregnancy (Dodd et al. 2014).

372

373 Acknowledgments

374 We thank the ARCH investigators and Comstock Lab members who were not

375 directly involved in the preparation of this manuscript but without whom this project

376 could not have been completed. 
378

379

380

381

382

383

384

385

386

387

388

389

390

391

392

393

394

395

396

397

398

399

400

401

402

403

404

405

406

407

408

409

410

411

412

413

414

415

416

417

418

419

420

421

Arimond M, Wiesmann D, Becquey E, Carriquiry A, Daniels MC, Deitchler M, FanouFogny N, Joseph ML, Kennedy G, Martin-Prevel Y, and Torheim LE. 2010. Simple food group diversity indicators predict micronutrient adequacy of women's diets in 5 diverse, resource-poor settings. J Nutr 140:2059S-2069S. 10.3945/jn.110.123414

Azadbakht L, and Esmaillzadeh A. 2011. Dietary diversity score is related to obesity and abdominal adiposity among Iranian female youth. Public Health Nutr 14:62-69. $10.1017 / \mathrm{S} 1368980010000522$

Black RE. 2001. Micronutrients in pregnancy. Br J Nutr 85 Suppl 2:S193-197.

Bolduc FV, Lau A, Rosenfelt CS, Langer S, Wang N, Smithson L, Lefebvre D, Alexander RT, Dickson CT, Li L, Becker AB, Subbarao P, Turvey SE, Pei J, Sears MR, Mandhane PJ, and Investigators CS. 2016. Cognitive Enhancement in Infants Associated with Increased Maternal Fruit Intake During Pregnancy: Results from a Birth Cohort Study with Validation in an Animal Model. Ebiomedicine 8:331-340. 10.1016/j.ebiom.2016.04.025

Borge TC, Aase H, Brantster AL, and Biele G. 2017. The importance of maternal diet quality during pregnancy on cognitive and behavioural outcomes in children: a systematic review and meta-analysis. Bmj Open 7. 10.1136/bmjopen-2017016777

Branum AM, Kirmeyer SE, and Gregory ECW. 2016. Prepregnancy Body Mass Index by Maternal Characteristics and State: Data From thte Birth Certificate, 2014. National Vital Statistics Reports: Centers for Disease Control and Prevention United State Department of Health and Human Services.

Chakona G, and Shackleton C. 2017. Minimum Dietary Diversity Scores for Women Indicate Micronutrient Adequacy and Food Insecurity Status in South African Towns. Nutrients 9. 10.3390/nu9080812

Chi SH, Grigsby-Toussaint DS, Bradford N, and Choi J. 2013. Can Geographically Weighted Regression improve our contextual understanding of obesity in the US? Findings from the USDA Food Atlas. Applied Geography 44:134-142. 10.1016/j.apgeog.2013.07.017

Costa MM, Belo S, Souteiro P, Neves JS, Magalhaes D, Silva RB, Oliveira SC, Freitas P, Varela A, Queiros J, and Carvalho D. 2018. Pregnancy after bariatric surgery: Maternal and fetal outcomes of 39 pregnancies and a literature review. J Obstet Gynaecol Res. 10.1111/jog.13574

DHHS, and USDA. 2015. 2015 - 2020 Dietary Guidelines for Americans. 8th Edition. U.S. Department of Health and Human Services; U.S. Department of Agriculture. Dodd JM, Cramp C, Sui ZX, Yelland LN, Deussen AR, Grivell RM, Moran LJ, Crowther CA, Turnbull D, McPhee AJ, Wittert G, Owens JA, Robinson JS, and Grp LRT. 2014. The effects of antenatal dietary and lifestyle advice for women who are overweight or obese on maternal diet and physical activity: the LIMIT randomised trial. Bmc Medicine 12. 10.1186/S12916-014-0161-Y

Englund-Ogge L, Brantsaeter AL, Haugen M, Sengpiel V, Khatibi A, Myhre R, Myking S, Meltzer HM, Kacerovsky M, Nilsen RM, and Jacobsson B. 2012. Association between intake of artificially sweetened and sugar-sweetened beverages and 
422

423

424

425

426

427

428

429

430

431

432

433

434

435

436

437

438

439

440

441

442

443

444

445

446

447

448

449

450

451

452

453

454

455

456

457

458

459

460

461

462

463

464

465

466

preterm delivery: a large prospective cohort study. Am J Clin Nutr 96:552-559. 10.3945/ajcn.111.031567

FAO, and 360 F. 2016. Minimum Dietary Diversity for Women: A Guide for Measurement. Rome: FAO.

Flynn AC, Schneeberger C, Seed PT, Barr S, Poston L, and Goff LM. 2015. The Effects of the UK Pregnancies Better Eating and Activity Trial Intervention on Dietary Patterns in Obese Pregnant Women Participating in a Pilot Randomized Controlled Trial. Nutr Metab Insights 8:79-86. 10.4137/NMI.S29529

Garrow JS, and Webster J. 1985. Quetelet's index (W/H2) as a measure of fatness. Int $J$ Obes 9:147-153.

Gernand AD, Schulze KJ, Stewart CP, West KP, Jr., and Christian P. 2016. Micronutrient deficiencies in pregnancy worldwide: health effects and prevention. Nat Rev Endocrinol 12:274-289. 10.1038/nrendo.2016.37

Godfrey KM, Reynolds RM, Prescott SL, Nyirenda M, Jaddoe VW, Eriksson JG, and Broekman BF. 2017. Influence of maternal obesity on the long-term health of offspring. Lancet Diabetes Endocrinol 5:53-64. 10.1016/S2213-8587(16)30107-3

Hanson MA, Bardsley A, De-Regil LM, Moore SE, Oken E, Poston L, Ma RC, McAuliffe FM, Maleta K, Purandare CN, Yajnik CS, Rushwan H, and Morris JL. 2015. The International Federation of Gynecology and Obstetrics (FIGO) recommendations on adolescent, preconception, and maternal nutrition: "Think Nutrition First". International Journal of Gynecology \& Obstetrics 131:S213-S253.

Hu FB. 2002. Dietary pattern analysis: a new direction in nutritional epidemiology. Curr Opin Lipidol 13:3-9.

Imamura F, Micha R, Khatibzadeh S, Fahimi S, Shi P, Powles J, Mozaffarian D, Global Burden of Diseases N, and Chronic Diseases Expert G. 2015. Dietary quality among men and women in 187 countries in 1990 and 2010: a systematic assessment. Lancet Glob Health 3:e132-142. 10.1016/S2214-109X(14)70381-X

Kiboi W, Kimiywe J, and Chege P. 2017. Determinants of dietary diversity among pregnant women in Laikipia County, Kenya: a cross-sectional study. BMC Nutrition 3. 10.1186/s40795-017-0126-6

Krzizek EC, Brix JM, Herz CT, Kopp HP, Schernthaner GH, Schernthaner G, and Ludvik B. 2017. Prevalence of Micronutrient Deficiency in Patients with Morbid Obesity Before Bariatric Surgery. Obes Surg. 10.1007/s11695-017-2902-4

Laraia BA, Bodnar LM, and Siega-Riz AM. 2007. Pregravid body mass index is negatively associated with diet quality during pregnancy. Public Health Nutr 10:920-926. 10.1017/S1368980007657991

Liese AD, Krebs-Smith SM, Subar AF, George SM, Harmon BE, Neuhouser ML, Boushey CJ, Schap TE, and Reedy J. 2015. The Dietary Patterns Methods Project: synthesis of findings across cohorts and relevance to dietary guidance. $J$ Nutr 145:393-402. 10.3945/jn.114.205336

Loy SL, Marhazlina M, Azwany YN, and Jan JMH. 2011. Higher Intake of Fruits and Vegetables in Pregnancy Is Associated with Birth Size. Southeast Asian Journal of Tropical Medicine and Public Health 42:1214-1223.

Malik VS, Schulze MB, and Hu FB. 2006. Intake of sugar-sweetened beverages and weight gain: a systematic review. Am J Clin Nutr 84:274-288. 
467

468

469

470

471

472

473

474

475

476

477

478

479

480

481

482

483

484

485

486

487

488

489

490

491

492

493

494

495

496

497

498

499

500

501

502

503

504

505

506

507

508

509

510

Marquez BVY. 2012. Association between sugar-sweetened beverages and both gestational weight gain and gestational diabetes M.S. The University of Texas.

Martin-Prevel Y, Allemand P, Wiesmann D, Arimond M, Ballard T, Deitchler M, Dop MC, Kennedy G, Lee WTK, and Moursi M. 2015. Moving forward on choosing a standard operational indicator of women's dietary diversity. Rome, Italy: Food and Agriculture Organization (FAO).

Mohd-Shukri NA, Duncan A, Denison FC, Forbes S, Walker BR, Norman JE, and Reynolds RM. 2015. Health Behaviours during Pregnancy in Women with Very Severe Obesity. Nutrients 7:8431-8443. 10.3390/nu7105403

$\mathrm{NIH}$. 1998. Clinical Guidlines on the Indentification, Evaluation, and Treatment of Overweight and Obesity in Adults: The Evidence Report. Bethesda, MD: National Institutes of Health; National Heart, Lung, and Blood Institute in cooperation with the National Institute of Diabetes and Digestive and Kidney Diseases.

Oestreich AK, and Moley KH. 2017. Developmental and Transmittable Origins of Obesity-Associated Health Disorders. Trends Genet 33:399-407. 10.1016/j.tig.2017.03.008

Ojha S, Robinson L, Symonds ME, and Budge H. 2013. Suboptimal maternal nutrition affects offspring health in adult life. Early Human Development 89:909-913.

Park S, McGuire LC, and Galuska DA. 2015. Regional Differences in Sugar-Sweetened Beverage Intake among US Adults. Journal of the Academy of Nutrition and Dietetics 115:1996-2002.

Poston L, Caleyachetty R, Cnattingius S, Corvalan C, Uauy R, Herring S, and Gillman MW. 2016. Preconceptional and maternal obesity: epidemiology and health consequences. Lancet Diabetes Endocrinol 4:1025-1036. 10.1016/S22138587(16)30217-0

Prevention CfDCa. 2018. National Center for Chronic Disease Prevention and Health Promotion, Division of Nutrition, Physical Activity, and Obesity. Data, Trend and Maps [online]. Centers for Disease Control and Prevention.

Shapiro ALB, Kaar JL, Crume TL, Starling AP, Siega-Riz AM, Ringham BM, Glueck DH, Norris JM, Barbour LA, Friedman JE, and Dabelea D. 2016. Maternal diet quality in pregnancy and neonatal adiposity: the Healthy Start Study. Int J Obes (Lond) 40:1056-1062. 10.1038/ijo.2016.79

Shin D, Chung H, Weatherspoon L, and Song WO. 2014. Validity of prepregnancy weight status estimated from self-reported height and weight. Matern Child Health J 18:1667-1674. 10.1007/s10995-013-1407-6

Shin D, Lee KW, and Song WO. 2016. Pre-Pregnancy Weight Status Is Associated with Diet Quality and Nutritional Biomarkers during Pregnancy. Nutrients 8:162. 10.3390/nu8030162

Stankowiak-Kulpa H, Kargulewicz A, Styszynski A, Swora-Cwynar E, and Grzymislawski M. 2017. Iron status in obese women. Ann Agric Environ Med 24:587-591. 10.5604/12321966.1232092

Sullivan KM, Ford ES, Azrak MF, and Mokdad AH. 2009. Multivitamin use in pregnant and nonpregnant women: results from the Behavioral Risk Factor Surveillance System. Public Health Rep 124:384-390. 10.1177/003335490912400307 
511 Tsigga M, Filis V, Hatzopoulou K, Kotzamanidis C, and Grammatikopoulou MG. 2011.

512

513

514

515

516

517

518

519

520

521

522

523

524

525

526

527

528

529

530
Healthy Eating Index during pregnancy according to pre-gravid and gravid weight status. Public Health Nutr 14:290-296. 10.1017/S1368980010001989

Vargas CM, Stines EM, and Granado HS. 2017. Health-equity issues related to childhood obesity: a scoping review. J Public Health Dent 77 Suppl 1:S32-S42. 10.1111/jphd.12233

Williams EP, Mesidor M, Winters K, Dubbert PM, and Wyatt SB. 2015. Overweight and Obesity: Prevalence, Consequences, and Causes of a Growing Public Health Problem. Curr Obes Rep 4:363-370. 10.1007/s13679-015-0169-4

Wolongevicz DM, Zhu L, Pencina MJ, Kimokoti RW, Newby PK, D'Agostino RB, and Millen BE. 2010. Diet quality and obesity in women: the Framingham Nutrition Studies. Br J Nutr 103:1223-1229. 10.1017/S0007114509992893

Xi B, Huang Y, Reilly KH, Li S, Zheng R, Barrio-Lopez MT, Martinez-Gonzalez MA, and Zhou D. 2015. Sugar-sweetened beverages and risk of hypertension and CVD: a dose-response meta-analysis. Br J Nutr 113:709-717. 10.1017/S0007114514004383

Zerfu TA, and Ayele HT. 2013. Micronutrients and pregnancy; effect of supplementation on pregnancy and pregnancy outcomes: a systematic review. Nutrition Journal 12. 


\section{Table $\mathbf{1}$ (on next page)}

Participant characteristics by study. ${ }^{1}$

${ }^{1}$ Comparisons across categories (i.e. normal, overweight, obese) within a characteristic (i.e. BMI) were compared between study cohorts using Fisher's exact test with $p$ values reported next to characteristic names. Proportions within each category (i.e. normal) of each characteristic were compared between study cohorts using Fisher's exact test with p-values reported in the far right column. 
1 Table 1. Participant Characteristics by Study. ${ }^{1}$

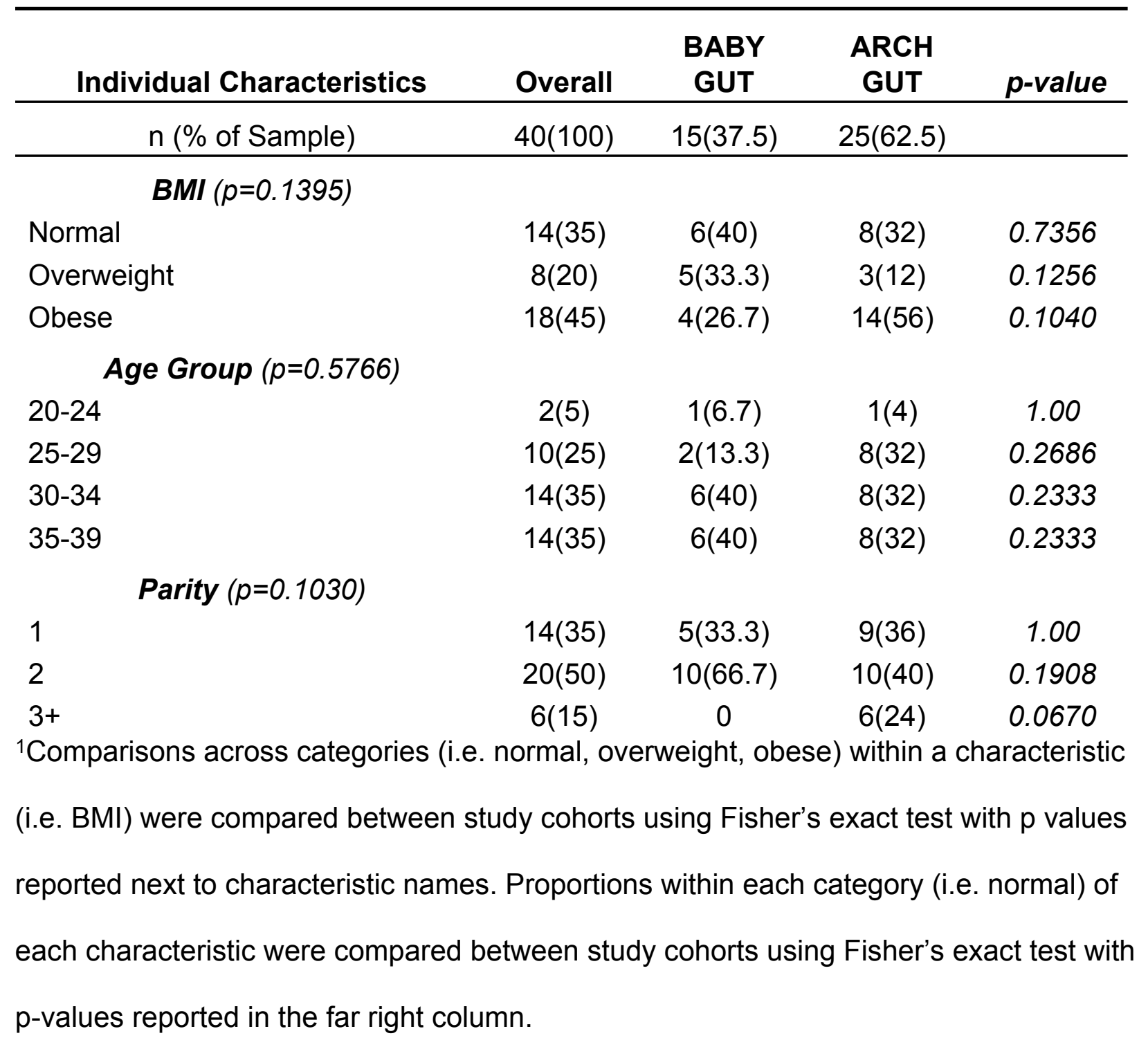




\section{Table 2 (on next page)}

MDD-W food group consumption according to BMI category and total MDD-W score.

Values indicate percent within BMI category who received the corresponding overall MDD-W score and consumed that particular category of food. * indicates that individuals consuming fewer than 5 food groups were less likely to have consumed a particular food group than individuals consuming equal to or more than 5 food groups. Overall comparisons of intake between individuals consuming fewer than 5 food groups compared to those consuming equal to or more than 5 food groups were tested using Fisher's exact test. 
1 Table 2. MDD-W food group consumption according to BMI category and total MDD-W score. Values indicate percent

2 within BMI category who received the corresponding overall MDD-W score and consumed that particular category of food.

\begin{tabular}{|c|c|c|c|c|c|c|c|c|}
\hline \multirow{3}{*}{$\begin{array}{l}\text { Sample Size (\%) } \\
\text { Sample Size (n) }\end{array}$} & \multirow{2}{*}{\multicolumn{2}{|c|}{$\frac{\text { Normal Weight }}{35}$}} & \multirow{2}{*}{\multicolumn{2}{|c|}{$\frac{\text { Overweight }}{20}$}} & \multirow{2}{*}{\multicolumn{2}{|c|}{ Obese }} & \multirow{2}{*}{\multicolumn{2}{|c|}{$\frac{\text { Overall }}{100}$}} \\
\hline & & & & & & & & \\
\hline & 2 & 12 & 1 & 7 & 11 & 7 & 14 & 26 \\
\hline MDD-W Score & $<5$ & $\geq 5$ & $<5$ & $\geq 5$ & $<5$ & $\geq 5$ & $<5$ & $\geq 5$ \\
\hline Grains & 100 & 100 & 100 & 100 & 90.9 & 100 & 92.9 & 100 \\
\hline Pulses (brans, peas, and lentils) & 0 & 16.7 & 0 & 28.6 & 0 & 14.3 & 0 & 19.2 \\
\hline Nuts and seeds & 0 & 41.7 & 0 & 42.9 & 9.1 & 28.6 & 7.1 & 38.5 \\
\hline Dairy & 100 & 91.7 & 0 & 85.7 & 81.8 & 85.7 & 78.6 & 88.5 \\
\hline Meat, fish, or poultry & 50 & 91.7 & 100 & 85.7 & 81.8 & 85.7 & 78.6 & 88.5 \\
\hline Eggs & 0 & 33.3 & 100 & 28.6 & 9.1 & 42.9 & 14.3 & 34.6 \\
\hline Dark green leafy vegetables & 50 & 50 & 0 & 42.9 & 0 & 57.1 & 7.1 & $50^{*}$ \\
\hline Other Vitamin A rich fruits or vegetables & 0 & 33.3 & 0 & 28.6 & 0 & 71.4 & 0 & $42.3^{*}$ \\
\hline Other Vegetables & 50 & 75 & 0 & 57.1 & 27.3 & 57.1 & 28.6 & $65.4^{*}$ \\
\hline Other fruits & 0 & 75 & 0 & 100 & 9.1 & 71.4 & 7.1 & $80.8^{*}$ \\
\hline
\end{tabular}

* indicates that individuals consuming fewer than 5 food groups were less likely to have consumed a particular food group than individuals consuming equal to or more than 5 food groups. Overall comparisons of intake between individuals consuming fewer than 5 food groups compared to those consuming equal to or more than 5 food groups were tested using Fisher's exact test. 
Figure 1

Pre-pregnancy BMI and MDD-W score during the third trimester of pregnancy are inversely correlated.

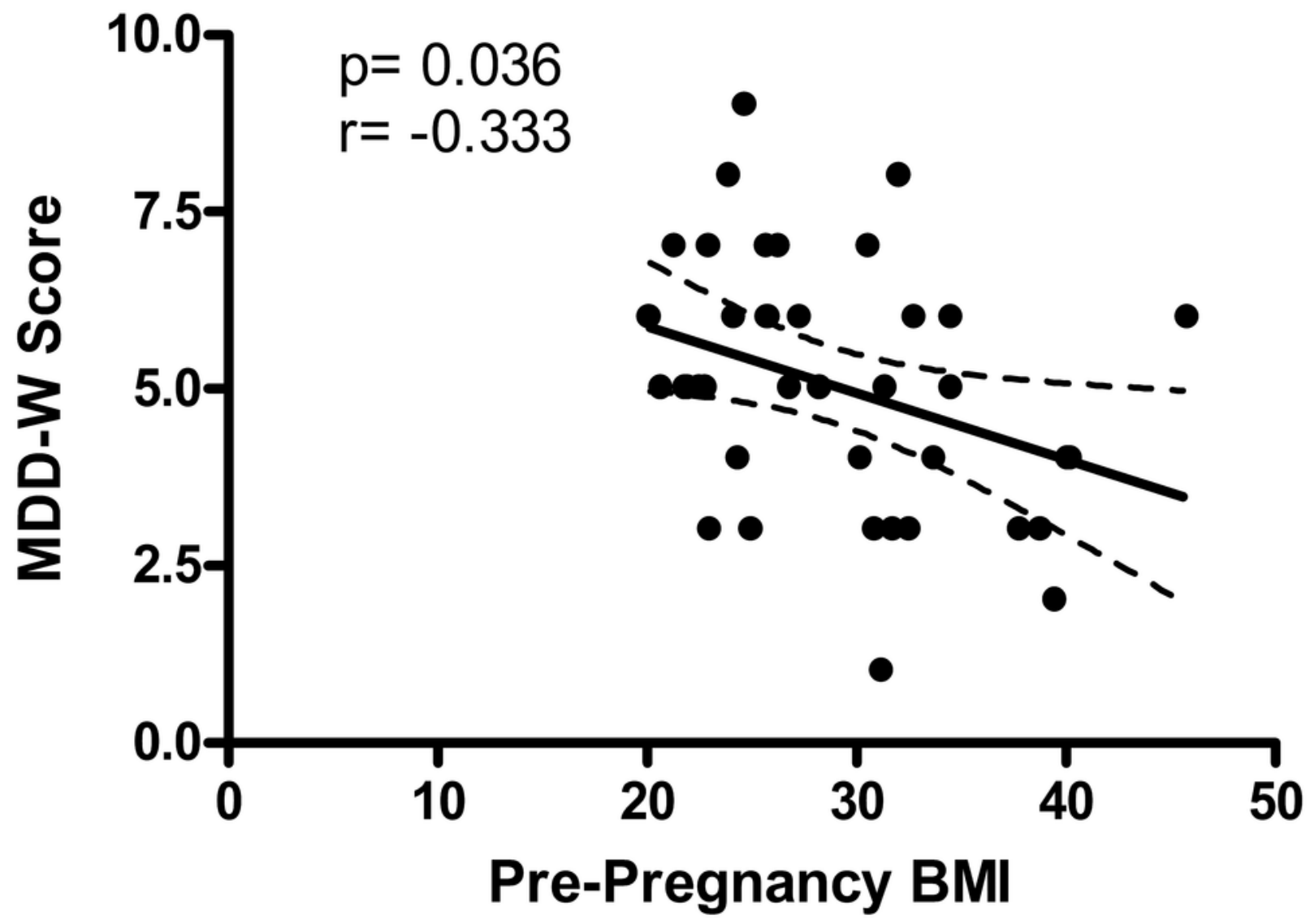


Figure 2 (on next page)

A smaller percentage of obese women received MDD-W scores of $\geq 5$ than did normal weight $(p=0.012)$ or overweight women $(p=0.036)$.

The percent of participants achieving a total MDD-W score $\geq 5$ differed by BMI category with $85.7 \%$ of normal weight women, $87.5 \%$ of overweight women, and $38.9 \%$ of obese women attaining MDD-W scores $\geq 5(p=0.007)$. Bars without a common superscript letter differ, $p \leq 0.05$. 


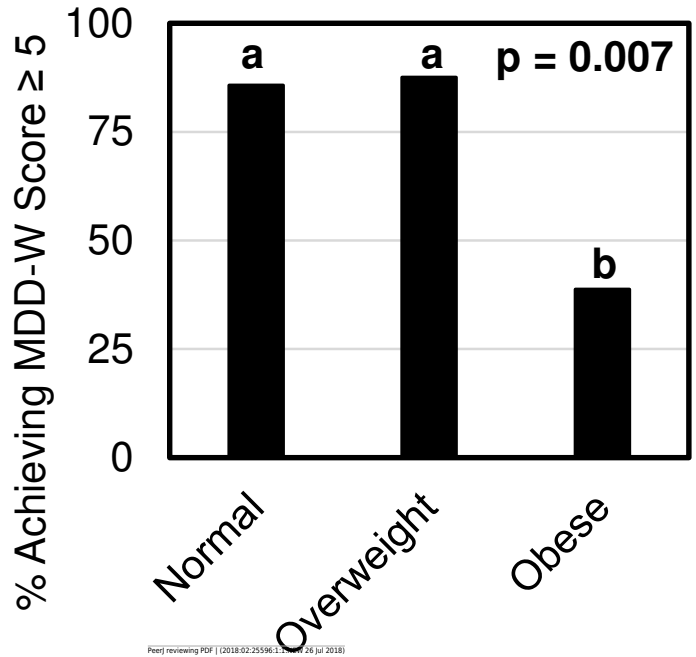


Figure 3

Third trimester MDD-W scores differed across the three pre-pregnancy BMI categories with no pairwise comparisons being significant.

The median MDD-W score is indicated by a horizontal line. parisons being significant.

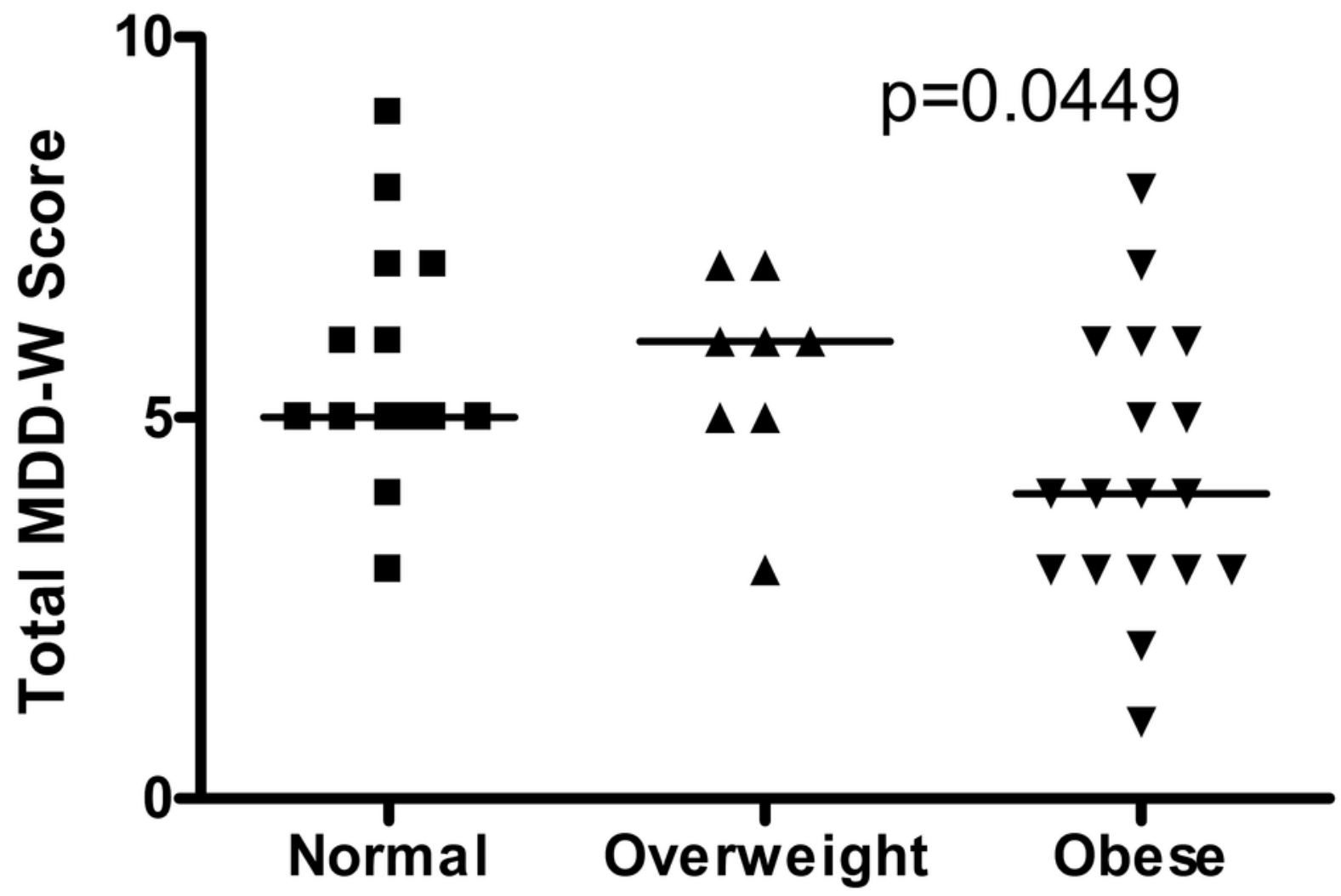




\section{Figure 4(on next page)}

Percent of pregnant women consuming specific food groups with low nutrient densities during the third trimester of pregnancy.

Sweetened drinks were consumed by $75 \%$ of pregnant women. Intake of these food groups did not differ by BMI category or study population. 


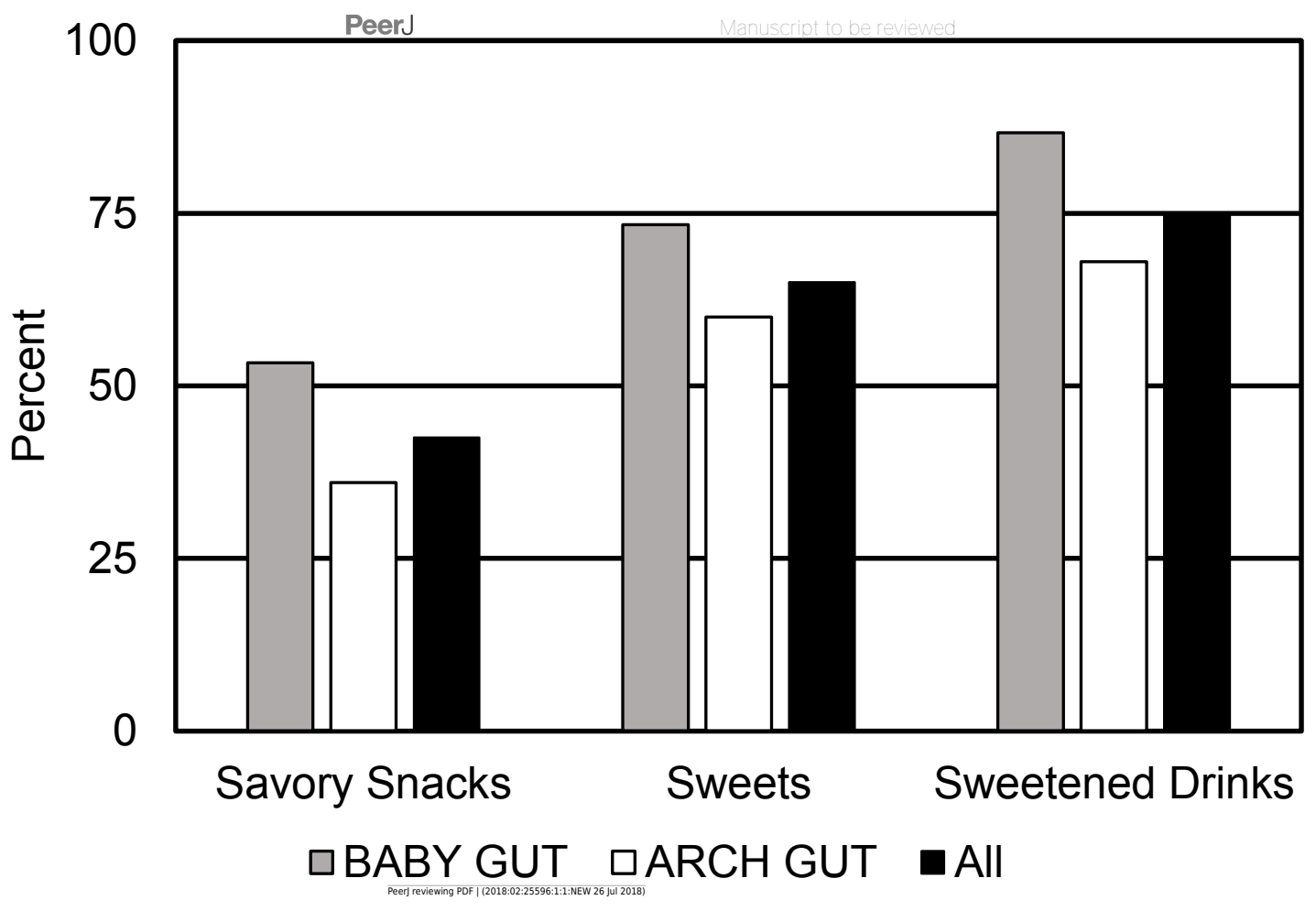

